\title{
Therapeutic Effects of Topical Tranexamic Acid in Comparison with Hydroquinone in Treatment of Women with Melasma
}

\author{
Najmolsadat Atefi - Behzad Dalvand - Mahammadreza Ghassemi • \\ Golnaz Mehran · Amir Heydarian
}

Received: May 15, 2017 / Published online: July 26, 2017

(C) The Author(s) 2017. This article is an open access publication

\begin{abstract}
Introduction: Few studies have focused on therapeutic as well as side effects of tranexamic acid (TXA) as a topical drug compared to other topical drugs in treating melasma. The present study aimed to assess and compare the beneficial therapeutic effects and also side effects of local TXA in comparison with hydroquinone in treating women with melasma.

Methods: This randomized double-blinded clinical trial was performed on 60 women who suffered from melasma and were referred to the skin disorders clinic at the Rasoul-e-Akram hospital in Tehran in 2015. The patients were then randomly assigned via computerized randomization to two groups: group A received TXA\%5 (topically twice a day for 12 weeks in the location of the melasma) and group B (received hydroquinone $2 \%$ with the same treatment order). Prior to intervention and at 12 weeks after intervention, the intensity and extension of melasma were assessed based on
\end{abstract}

Enhanced content To view enhanced content for this article go to www.medengine.com/Redeem/ 23E8F06014644CF7.

N. Atefi · B. Dalvand $(\bowtie) \cdot$ M. Ghassemi ·

G. Mehran · A. Heydarian

Dermatology Department, Rasoul-e-Akram Hospital, Iran University of Medical Science, Tehran, Iran e-mail: Hosna.rcrdc@gmail.com the Melasma Area and Severity Index (MASI) scoring method.

Results: The mean MASI score in both treatment groups decreased considerably after completion of treatment and was not significant between the two groups. No side effects were detected in group A, but $10 \%$ of those in group B complained of drug-related side effects including erythema and skin irritation $(p=0.131)$. Regarding the level of patient satisfaction, the patients in group A had a significantly higher level of satisfaction level of 33.3\% compared with $6.7 \%$ in group B $(p=0.015)$ (Fig. 9). Multivariate linear regression modeling with the presence of age, history of systemic disorder, drug history, and family history of melasma demonstrated no difference in the mean MASI between the two groups.

Conclusion: Topical use of TXA significantly reduced both melanin level and MASI score. Given its high efficiency and low drug side effects, this regimen results in high patient satisfaction compared with topical hydroquinone. IRCT code: IRCT2016040627220N2.

Keywords: Hydroquinone;

Melasma; Tranexamic acid; Women

\section{INTRODUCTION}

Melasma is a global melanogenesis dysfunction derived from hypermelanosis of the skin due to 
various underlying risk factors, including sun exposure, hormonal changes, pregnancy, genetic predisposition, combined oral contraceptives, use of cosmetics and photosensitizing drugs, hepatic disorders, and inflammatory processes [1-3]. Epidemiologically, this condition is observed in all geographical and ethnical subgroups, but it occurs with a higher tendency in more pigmented phenotypes, such as East Asians, Middle Easterners, and Mediterranean Africans [4]. The overall incidence of melasma remains unclear, but it presents an upward trend because of increased sun exposure time during leisure and daily activities, potential genetic polymorphisms due to industrialized mutagens, increased post-inflammatory hyperpigmentation, and some sex-related hormonal disturbances $[5,6]$. Pathophysiologically, melasma is sourced from the tripartite intersection among genetic susceptibility, environmental factors, and hormonal changes. This interaction can result in melanogenic hyperactivity and the development of epidermal hyperpigmentation [7]. Diagnostic protocols and therapeutic approaches are based on determining the intensity and extension of hyperpigmentation along with prevention of this process. Both palliative and invasive methods have been conducted to treat melasma and prevent its development. Among noninvasive methods, the use of topical sunscreens and drugs is superior to the use of other agents [7] in controlling the intensity and extension of hyperpigmentation and for prevention of this process. Sunscreens can prevent hyperpigmentation and relapse of melasma. Among topical and systemic drugs, hydroquinone is extensively used because of its potential effects on inhibiting the conversion of dopa to melanin by blocking tyrosinase action and suppressing the formation, melanization, and degradation of melanosomes $[8,9]$.

Among other therapies, the use of tranexamic acid (TXA) has been recently recommended due to its effects of decreasing melanogenesis in epidermal melanocytes and providing rapid and sustained lightening $[10,11]$. However, few studies have focused on the therapeutic and side effects of TXA as a topical drug compared with other topical drugs.
This study aimed to assess and compare the beneficial therapeutic effects as well as the side effects of local TXA in comparison with hydroquinone in treating women with melasma.

\section{METHODS}

This randomized double-blinded clinical trial was performed on 60 women who suffered from melasma and were referred to the skin disorder clinic at the Rasoul-e-Akram Hospital in Tehran in 2015. Women aged lower than 18 years, from west Asia, and those with a recent history of chronic systemic disorders, history of using anti-pigmentation drugs, or history of treating melasma within 6 months prior to the study were all excluded. This study was approved by the Ethics Committee of Iran University of Medical Sciences. Informed consent was obtained from the patients. Human rights were respected in accordance with the Helsinki Declaration.

The patients were interviewed to collect demographic and clinical data. The women were also completely examined to assess evidence of skin and physical disorders. The patients were randomly assigned via computerized randomization into two groups: group A (received TXA5\% topically twice a day for 12 weeks in the location of the melasma) and group B (received hydroquinone $2 \%$ with the same treatment order). Neither patient nor physician were aware of the nature of the ordered drugs. Both groups used one type of sunscreen with SPF30. Before intervention and at 12 weeks after intervention, the intensity and extension of melasma were assessed based on the Melasma Area and Severity Index (MASI) scoring method. The details of this system have been previously described. In brief, the MASI score was calculated according to the following formula:

MASI $=$ extension of darkening $\times$ (intensity of darkening or degree of pigmentation + homogeneity of darkening).

The extension of darkening was scored on the basis of the area of melasma involvement from 0 to 6 , in which $0=$ no involvement, $1=$ less than $10 \%$ involvement, $2=10-29 \%$ involvement, $\quad 3=30-49 \%$ involvement, 
$4=50-69 \%$ involvement, $5=70-89 \%$ involvement, or $6=90-100 \%$ involvement. The degree of pigmentation and homogeneity (color uniformity) was graded from 0 to 4 as follows: $0=$ absent, $1=$ slight, $2=$ mild, $3=$ marked, or $4=$ maximum. MASI included $30 \%$ of involvement on the forehead, $30 \%$ on both left and right cheeks and $10 \%$ on the chin. Changes in the MASI score after treatment protocols and the level of patient satisfaction were assessed and rated as poor, intermediate, and high. Using the patients' comments, the apparent improvement in melasma after treatment was low, medium, or very good.

For statistical analysis, results were presented as the mean \pm standard deviation for quantitative variables and summarized by absolute frequencies and percentages for categorical variables. Data normality was analyzed using the Kolmogorov-Smirnoff test. Categorical variables were compared using the Chi square test or Fisher's exact test when more than 20\% of cells with an expected count of less than 5 were observed. Quantitative variables were also compared with $t$ tests or Mann-Whitney $U$ tests. The paired $t$ test or Wilcoxon test was applied to assess changes in the MASI score after intervention compared with baseline. Statistical software SPSS v.16.0 for Windows (SPSS; Chicago, IL, USA) was used for statistical analysis. Statistical significance was set at $p$ values of 0.05 or less.

\section{RESULTS}

Thirty patients received TXA as group A and 30 received hydroquinone as group $\mathrm{B}$. The two groups were matched for mean age $(38.10 \pm 6.27$ vs. $39.97 \pm 7.86$ years, $p=0.314)$, history of receiving systemic drugs (especially thyroid drugs) $(30.0 \%$ vs. $13.3 \%, p=0.117)$, history of systemic disorders $(13.3 \%$ vs. $6.7 \%$, $p=0.671)$, and family history of melasma (50.0\% vs. $46.7 \%, p=0.796)$. As shown in Table 1, with respect to the intensity and extension of disease in the two groups, the mean percentage of forehead involvement at baseline was higher in group A than in group B, whereas the areas of involvement in other locations, including left and right cheeks and chin, were similar between the groups at baseline. Similarly, no difference was found in the mean score of pigmentation and homogeneity of melasma between groups A and B before the intervention (Table 1). The mean MASI percentages at baseline in groups A and B were $72.43 \pm 20.64$ (Figs. 1, 2) and $65.93 \pm 18.11$ (Figs. 3, 4), respectively, with no difference between the two groups $(p=0.200)$. In addition, the mean MASI scores in groups $\mathrm{A}$ and $\mathrm{B}$ were $4.80 \pm 1.06$ and $4.37 \pm 0.93$, respectively, with no difference between the groups $(p=0.098)$. Table 1 illustrates the severity of melasma after treatment. The mean scores for area of involvement were significantly lower in group A than in group B, but the mean scores for degree of pigmentation and homogeneity were not different between the two groups after receiving the ordered drugs. The mean MASI percentage at the end of treatment was $26.60 \pm 13.43$ in group A (Figs. 5, 6) and $23.73 \pm 12.84$ in group B (Figs. 7,8 ) with no difference between the groups $(p=0.402)$. Moreover, the mean MASI scores in groups A and $B$ were $2.33 \pm 0.71$ and $2.30 \pm 0.65$, respectively, with no difference between the groups $(p=0.850)$. The mean MASI score in both groups significantly decreased after both treatment options compared with baseline assessment $(p<0.001)$. In group $\mathrm{A}$, no side effect was revealed, but $10 \%$ of those in group B suffered from drug-related side effects, including erythema and skin irritation $(p=0.131)$. Regarding the level of patient satisfaction (Fig. 9), the patients in group A had significantly higher level of satisfaction than those in group B, with high satisfaction level in $33.3 \%$ of patients in group A and only in $6.7 \%$ in group B $(p=0.015)$. In wood lamp examination, a positive result was found in $40 \%$ of cases in group A and $30 \%$ in group $\mathrm{B}$, and the difference between the two groups was no significant $(p=0.464)$. Multivariate linear regression modeling (Table 2) with the presence of age, history of systemic disorder, drug history, and family history of melasma revealed no difference in the mean MASI percentage between the two groups after completing the therapeutic regimens. In a similar regression model (Table 3 ), no difference 
Table 1 The change in melasma indexes before and after treatment methods

\begin{tabular}{|c|c|c|c|c|c|c|}
\hline \multirow[t]{2}{*}{ Item } & \multicolumn{2}{|c|}{ Before treatment } & \multirow[t]{2}{*}{$p$ value } & \multicolumn{2}{|c|}{ After treatment } & \multirow[t]{2}{*}{$p$ value } \\
\hline & Group TXA & $\overline{\text { Group HYD }}$ & & Group TXA & Group HYD & \\
\hline \multicolumn{7}{|l|}{ Extension } \\
\hline Forehead & $16.83 \pm 4.99$ & $12.57 \pm 3.71$ & 0.001 & $9.23 \pm 4.11$ & $6.83 \pm 2.56$ & 0.009 \\
\hline Left cheek & $17.17 \pm 4.85$ & $15.33 \pm 3.38$ & 0.095 & $9.90 \pm 4.22$ & $7.70 \pm 1.46$ & 0.017 \\
\hline Right cheek & $17.17 \pm 4.85$ & $15.33 \pm 3.38$ & 0.095 & $9.50 \pm 4.28$ & $7.70 \pm 2.46$ & 0.017 \\
\hline Chin & $5.07 \pm 1.72$ & $4.87 \pm 0.90$ & 0.575 & $2.57 \pm 1.16$ & $2.80 \pm 1.06$ & 0.421 \\
\hline Area & $4.20 \pm 0.76$ & $4.27 \pm 0.78$ & 0.780 & $2.57 \pm 0.89$ & $3.13 \pm 0.81$ & 0.013 \\
\hline Pigmentation & $2.53 \pm 0.51$ & $2.67 \pm 0.47$ & 0.300 & $1.47 \pm 0.52$ & $1.73 \pm 0.52$ & 0.052 \\
\hline Homogensity & $2.00 \pm 0.37$ & $2.17 \pm 0.38$ & 0.910 & $1.33 \pm 0.48$ & $1.43 \pm 0.57$ & 0.464 \\
\hline MASI percentage & $72.43 \pm 20.64$ & $65.93 \pm 18.11$ & 0.200 & $26.60 \pm 13.43$ & $23.73 \pm 12.84$ & 0.402 \\
\hline MASI score & $4.80 \pm 1.06$ & $4.37 \pm 0.93$ & 0.098 & $2.33 \pm 0.71$ & $2.30 \pm 0.65$ & 0.850 \\
\hline
\end{tabular}

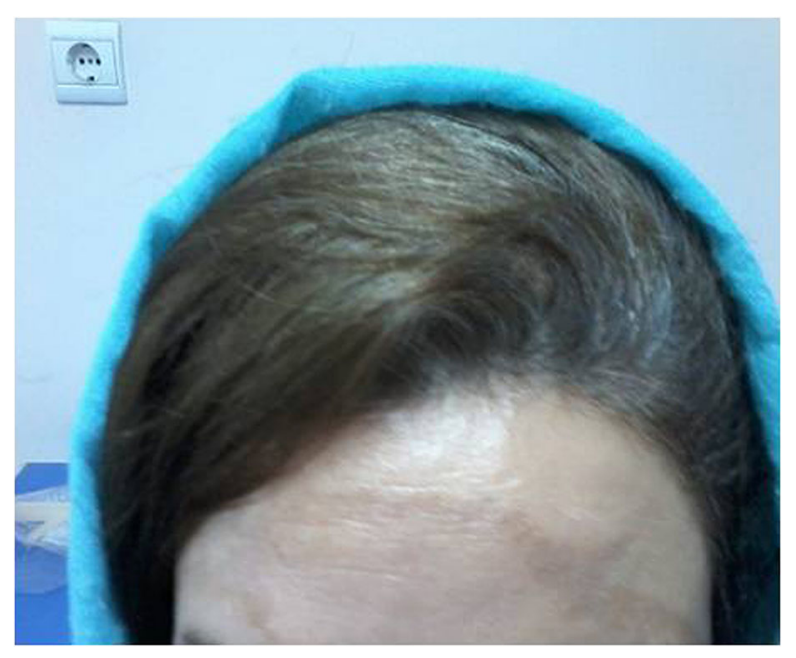

Fig. 1 A. Pretreatment TAX

was demonstrated in the mean total MASI between the groups after adjusting baseline variables.

\section{DISCUSSION}

According to the pathophysiological basis of melasma, various methods have been introduced to treat this disorder. However, most methods, especially topical noninvasive types,

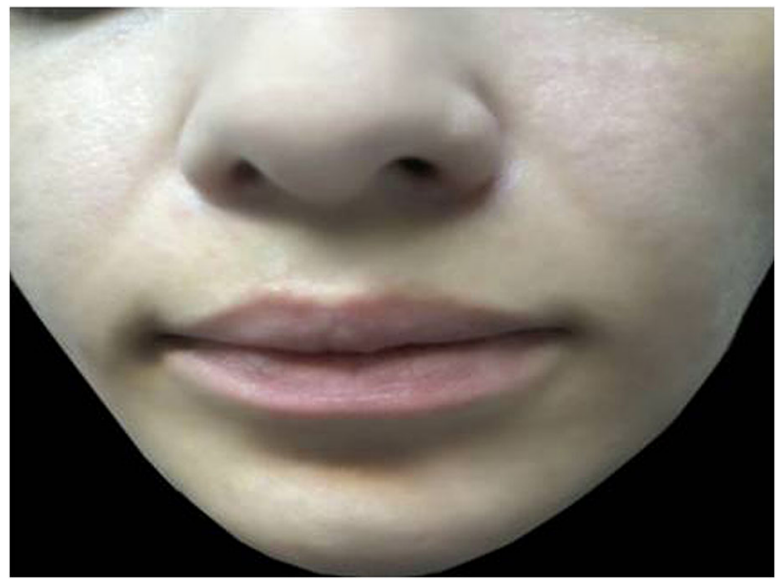

Fig. 2 B. Pretreatment TAX

are accompanied by partial therapeutic responses. This study attempted to assess treatment effects of TXA in comparison with hydroquinone in preventing the extension and deterioration of disease symptoms, as well as the level of patient satisfaction. Our results showed similar changes in disease extension and integrity after a 12-week intervention by the two drug regimens. However, a low complication rate in the group receiving TXA could lead to the high patient satisfaction, while the prescription of hydroquinone led to skin irritation 


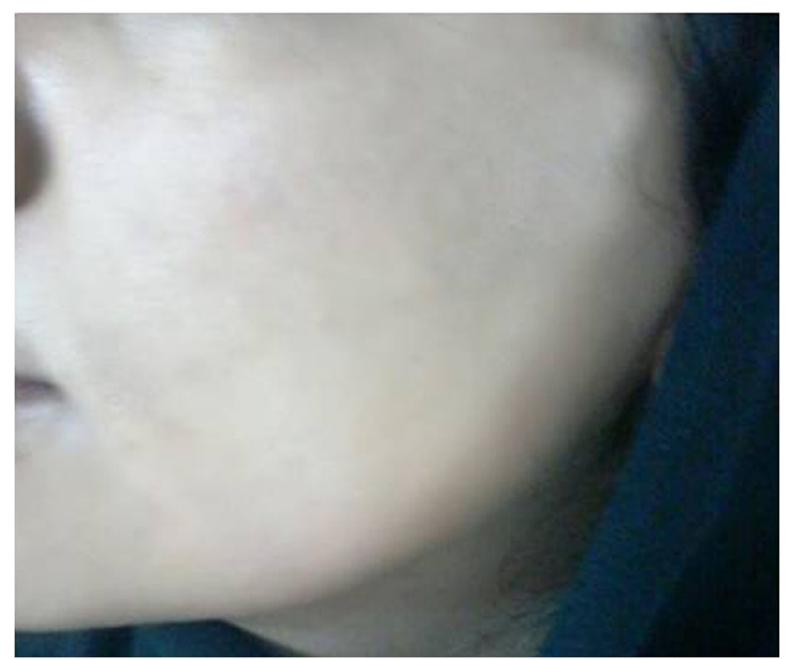

Fig. 3 C. Pretreatment HYD

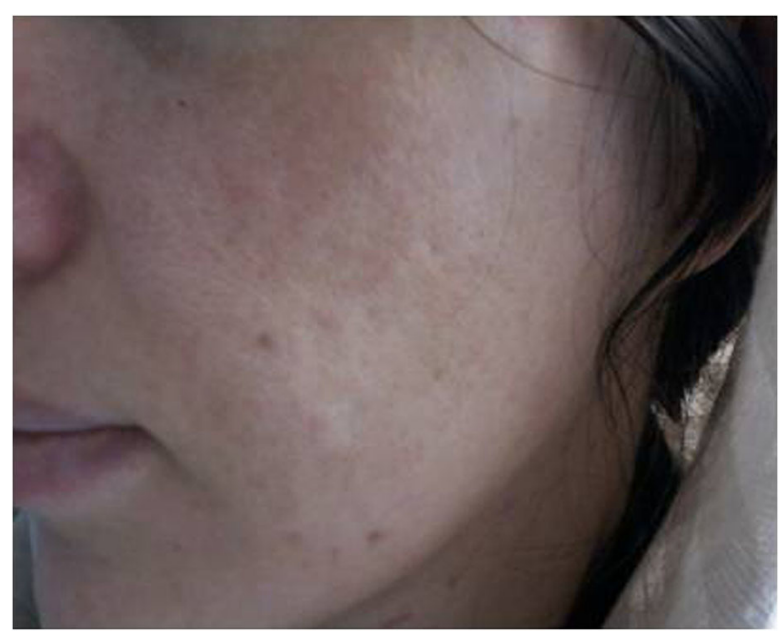

Fig. 4 D. Preteatment HYD

and erythema in $10 \%$ of cases, resulting in high dissatisfaction of treatment protocol.

Although most studies have focused on the high efficiency of systemic or oral use of TXA, its topical use has not been exclusively assessed. In a study by Kim et al. [12], the mean score of MASI considerably reduced following the use of topical TXA with a total response rate of $95.6 \%$. Further studies are necessary to determine the mechanisms of TXA in treating melasma. The ultraviolet irradiation exposure is crucial in the pathogenesis of melasma. UV irradiation induces plasminogen activator synthesis and

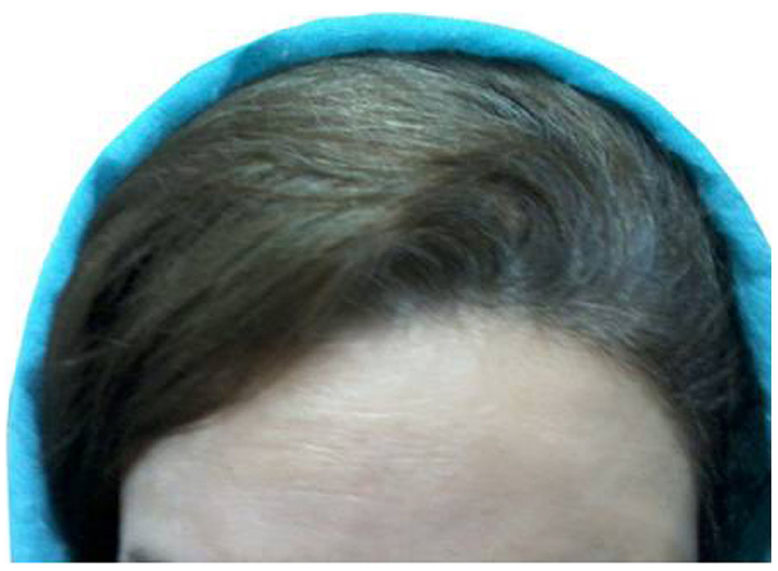

Fig. 5 A. Treatment TXA, after 12 weeks

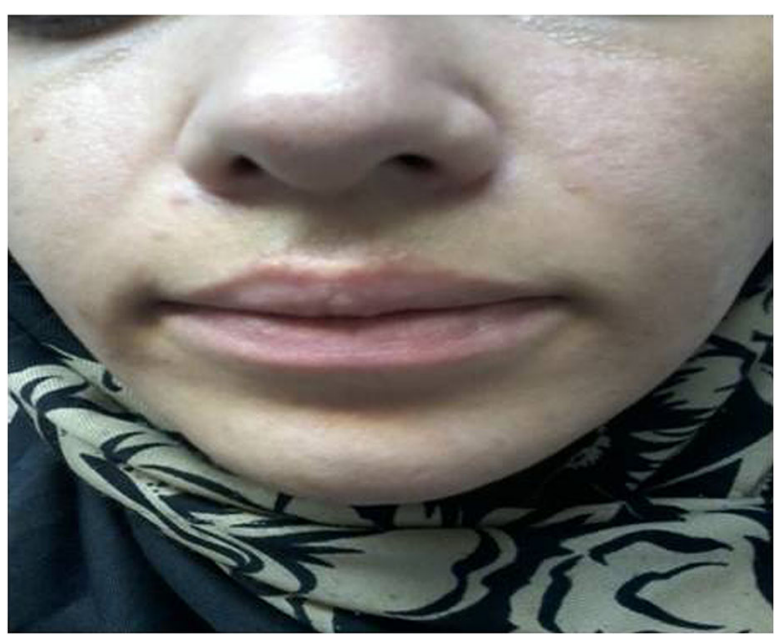

Fig. 6 B. Treatment TXA, after 12 weeks

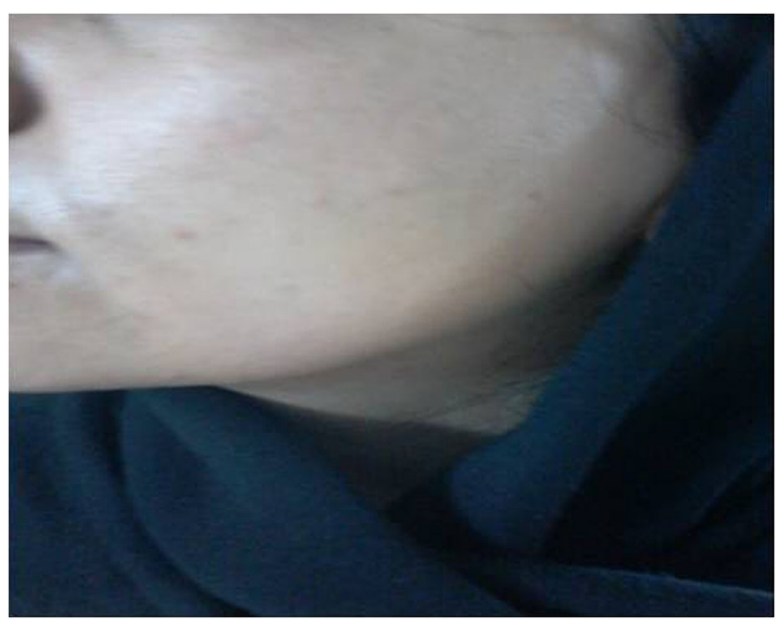

Fig. 7 C. Treatment HYD, after 12 weeks 


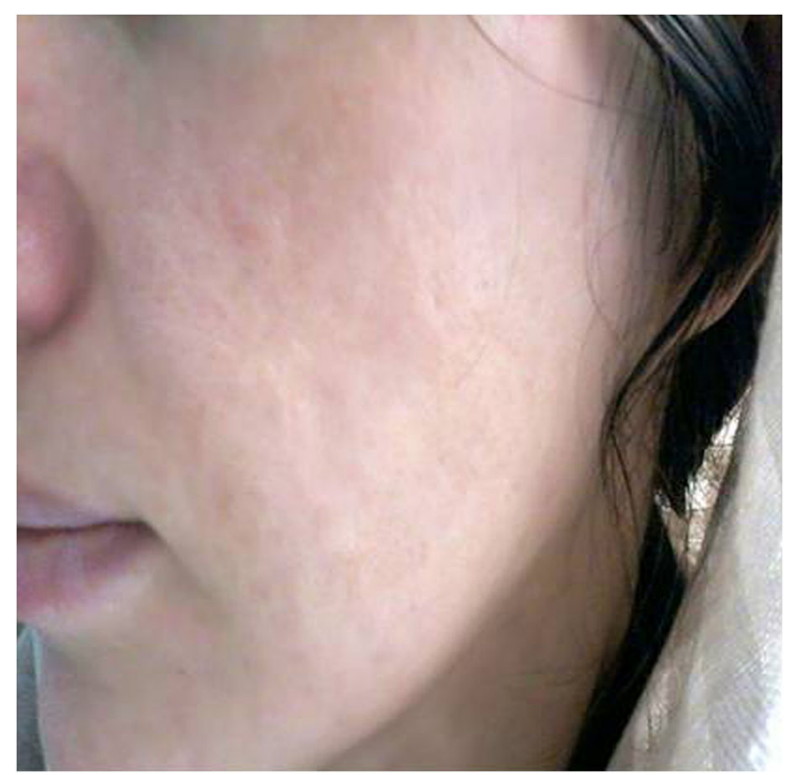

Fig. 8 D. Treatment HYD, after 12 weeks

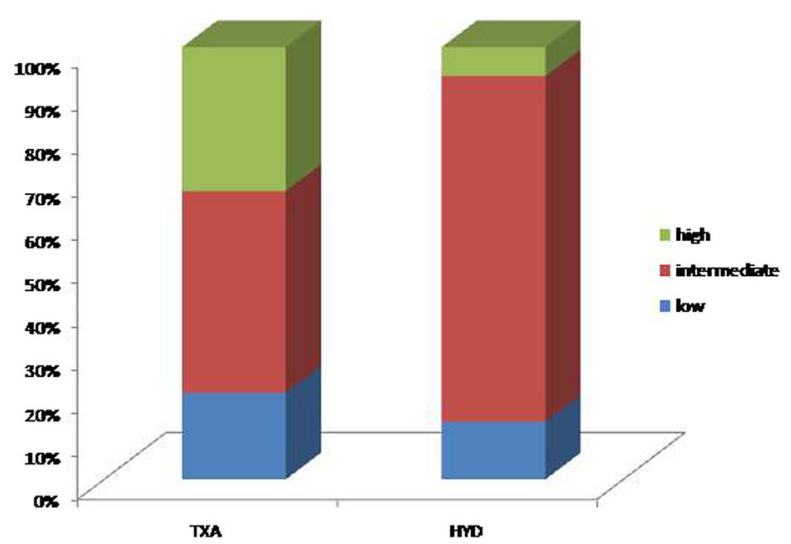

Fig. 9 The level of satisfaction of the two treatment regimens in melasma. $H Y D$ hydroquinone, $T X A$ tranexamic acid

increases plasmin activity in keratinocytes, thereby stimulating the release of arachidonic acid (AA) via phospholipase A2 free AA stimulates melanogenesisvia its metabolite, prostaglandinE2 (Fig. 9) [13].

The release of AA is increased by plasmin in endothelial cells. Increased plasmin elevates the melanocyte-stimulating hormone which activates melanin synthesis in melaniocytes. Plasmin is also crucial in the reiease of basic fibroblast growth factor (FGF) which is a potent melanocyte growth factor. All these processes increase melanin production in the skin (Fig. 10). Apart from its effect on melanocytes, plasmin plays an important role in angiogenesis. Plasmin converts extracellular matrix-bound VEGF into freely diffusible forms. TXA, a plasmin inhibitor, suppresses angiogenesis and neovascularization induced by basic fibroblast growth factor [14]. Single chain urokinase plasminogen activator (Se-uPA) in keratinocytes induces a dose-dependent increase in melanocytes tyrosinase activity and cell perimeter area and increased dendrites. Plasmin can significantly increase area and increased dendrites generated by keratinocytes elevate melanocyte activity.TXA prevents UV-induced pigmentation by interfering with the structure of plasminogen to the lysine binding sites of kerationytes. The consequences of such events is a reduction in free AA leading to a decreased ability to produce prostaglandins and a decline in melanocyte tyrosinase activity and melanogenesis [14]. TXA does not influence non-sun-exposed healthy skin. Moreover, action of TXA on angiogensis via plasmin also contributes to melasma. Blocking of the Sc-uPA pathway may be another mechanism through which TXA reduces hyperigmentation [14]. The effectiveness of TXA has been proven in several experimental and in vivo studies. Maeda and Tomitab [15] suggested that TXA inhibits melanin synthesis in melanocytes by interfering with the interaction of melanocytes and keratinocytes through inhibition of the plasminogen plasmin system. Meanwhile, Zhang et al. [16] showed that TXA can inhibit melanogenesis by interfering with the catalytic reaction of tyrosinase. Moreover, TXA is structurally similar to tyrosine which can competitively inhibit tyrosinase activity. 
Table 2 Multivariate linear regression analysis to assess the difference in the effect of treatment protocols on MASI percentage

\begin{tabular}{|c|c|c|c|c|c|}
\hline \multirow[t]{2}{*}{ Variable } & \multicolumn{2}{|c|}{ Unstandardized coefficients } & \multirow{2}{*}{$\begin{array}{l}\text { Standardized coefficients } \\
\text { Beta }\end{array}$} & \multirow[t]{2}{*}{$t$} & \multirow[t]{2}{*}{$p$ value } \\
\hline & Beta & SE & & & \\
\hline Constant & 48.854 & 22.020 & & 2.219 & 0.031 \\
\hline Group & 3.049 & 4.753 & 0.088 & 0.641 & 0.524 \\
\hline Age & -0.103 & 0.336 & -0.042 & -0.306 & 0.761 \\
\hline Drug history & -6.120 & 7.514 & -0.146 & -0.814 & 0.419 \\
\hline Disease history & 4.925 & 9.969 & 0.085 & 0.494 & 0.623 \\
\hline Melasma history & -2.537 & 4.801 & -0.073 & -0.528 & 0.599 \\
\hline
\end{tabular}

Table 3 Multivariate linear regression analysis to assess the difference in the effect of treatment protocols on MASI score

\begin{tabular}{|c|c|c|c|c|c|}
\hline \multirow[t]{2}{*}{ Variable } & \multicolumn{2}{|c|}{ Unstandardized coefficients } & \multirow{2}{*}{$\begin{array}{l}\text { Standardized coefficients } \\
\text { Beta }\end{array}$} & \multirow[t]{2}{*}{$t$} & \multirow[t]{2}{*}{$p$ value } \\
\hline & $\overline{\text { Beta }}$ & SE & & & \\
\hline Constant & 1.513 & 1.232 & & 1.228 & 0.225 \\
\hline Group & 0.371 & 0.266 & 0.189 & 1.394 & 0.169 \\
\hline Age & -0.001 & 0.019 & -0.008 & -0.063 & 0.950 \\
\hline Drug history & -0.393 & 0.420 & -0.165 & -0.936 & 0.353 \\
\hline Disease history & 0.527 & 0.558 & 0.161 & 0.946 & 0.348 \\
\hline Melasma history & -2.537 & 4.801 & -0.073 & -0.528 & 0.599 \\
\hline
\end{tabular}

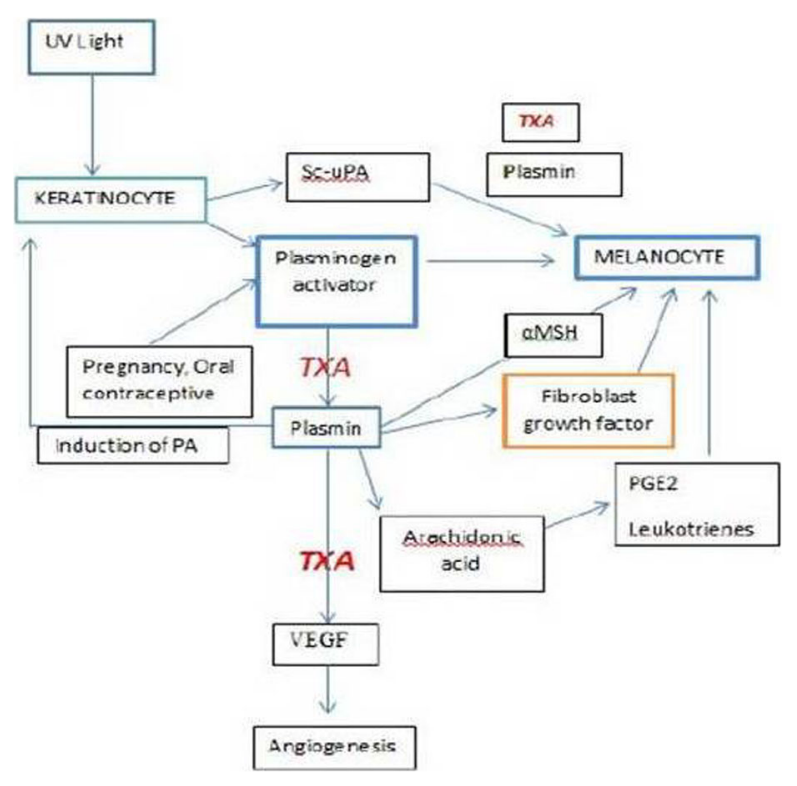

Fig. 10 Pathogenesis of melasma and role of tranexemic acid $(T X A)$, plasminogen activator $(P A)$, vascular endothelial growth factor $(V E G F)$

\section{CONCLUSIONS}

In conclusion, topical use of TXA can significantly reduce both melanin level and MASI score. This regimen results in high patient satisfaction because of its high efficiency and low drug side effects.

\section{ACKNOWLEDGEMENTS}

No funding or sponsorship was received for this study or publication of this article. The article processing charges were funded by the authors. All named authors meet the International Committee of Medical Journal Editors (ICMJE) criteria for authorship for this manuscript, take responsibility for the integrity of the work as a whole, and have given final approval for the version to be published. 
The authors wish to thank Rasoul-e-Akram Hospital Clinical Research Development Center for technically supported implementation of the project.

Compliance with Ethics Guidelines. This study was approved by the Ethics Committee of Iran University of Medical Sciences. Informed consent was obtained from the patients. Human rights were respected in accordance with the Helsinki Declaration.

Disclosures Najmolsadat Atefi, Behzad Dalvand, Mahammadreza Ghassemi, Golnaz Mehran and Amir Heydarian have nothing to disclose.

Open Access. This article is distributed under the terms of the Creative Commons Attribution-NonCommercial 4.0 International License (http://creativecommons.org/licenses/by-nc/4. $0 /$ ), which permits any noncommercial use, distribution, and reproduction in any medium, provided you give appropriate credit to the original author(s) and the source, provide a link to the Creative Commons license, and indicate if changes were made.

\section{REFERENCES}

1. Miot LD, Miot HA, Silva MG, Marques ME. Physiopathology of melasma. An Bras Dermatol. 2009;84:623-35.

2. Walker SL, Shah M, Hubbard VG, Pradhan HM, Ghimire M. Skin disease is common in rural Nepal: results of a point prevalence study. Br J Dermatol. $2008 ; 158: 334-8$.

3. Alakloby OM. Pattern of skin diseases in Eastern Saudi Arabia. Saudi Med J. 2005;26:1607-10.

4. Halder RM, Grimes PE, McLaurin CI, Kress MA, Kenney JA Jr. Incidence of common dermatoses in a predominantly black dermatologic practice. Cutis. 1983;32(388):390.
5. Perez M, Luke J, Rossi A. Melasma in Latin Americans. J Drugs Dermatol. 2011;10:517-23.

6. Sheth VM, Pandya AG. Melasma: a comprehensive update: part I. J Am AcadDermatol. 2011;65:689-97.

7. Tamega Ade A, Miot LD, Bonfietti C, Gige TC, Marques ME, Miot HA. Clinical patterns and epidemiological characteristics of facial melasma in Brazilian women. J Eur Acad Dermatol Venereol. 2013;27:151-6.

8. Katsambas A, Antoniou C. Melasma. Classification and treatment. J Eur Acad Dermatol Venereol. $1995 ; 4: 217-23$.

9. Lakhdar H, Zouhair K, Khadir K, et al. Evaluation of the effectiveness of a broad-spectrum sunscreen in the prevention of chloasma in pregnant women. J Eur Acad Dermatol Venereol. 2007;21:738-42.

10. Wu S, Shi H, Wu H, et al. Treatment of melasma with oral administration of tranexamic acid. Aesthetic Plast Surg. 2012;36(4):964-70.

11. Kanechorn $\mathrm{Na}$ Ayuthaya $\mathrm{P}$, Niumphradit $\mathrm{N}$, Manosroi A, Nakakes A. Topical 5\% tranexamic acid for the treatment of melasma in Asians: a double-blind randomized controlled clinical trial. J Cosmet Laser Ther. 2012; 14(3):150-154.

12. Kim SJ, ParkJY, Shibata T, Fujiwara R, Kang HY. Efficacy and possible mechanisms of topical tranexamic acid in melasma. Clin Exp Dermatol. 2016;41(5):480-5. doi:10.1111/ced.12835 (Epub 2016 May 2).

13. Maeda K, Naganuma M. Topical trans-4-aminomethyl cyclohexanecarboxylic acid prevents ultraviolet radiation-induced pigmentation. J Photochem Photobiol B. 1998;47:136-41.

14. Poojary S, Minni K. Tranxemic acid in melasma. J Pigment Disord. 2015;2:288.

15. Maeda K, Tomitab Y. Mechanism of the inhibitory effect of tranexamic acid on melanogenesis in cultured human melanocytes in the presence of keratinocyte-conditioned medium. J Health Sci. 2007;53:389-96.

16. Zhang X, Yang X, Yang H, Yang Y. Study of inhibitory effect of acidum tranexamicum on melanin synthesis. Chin J Dermatovenereol Integr Tradit West Med. 2003;2:227-9. 